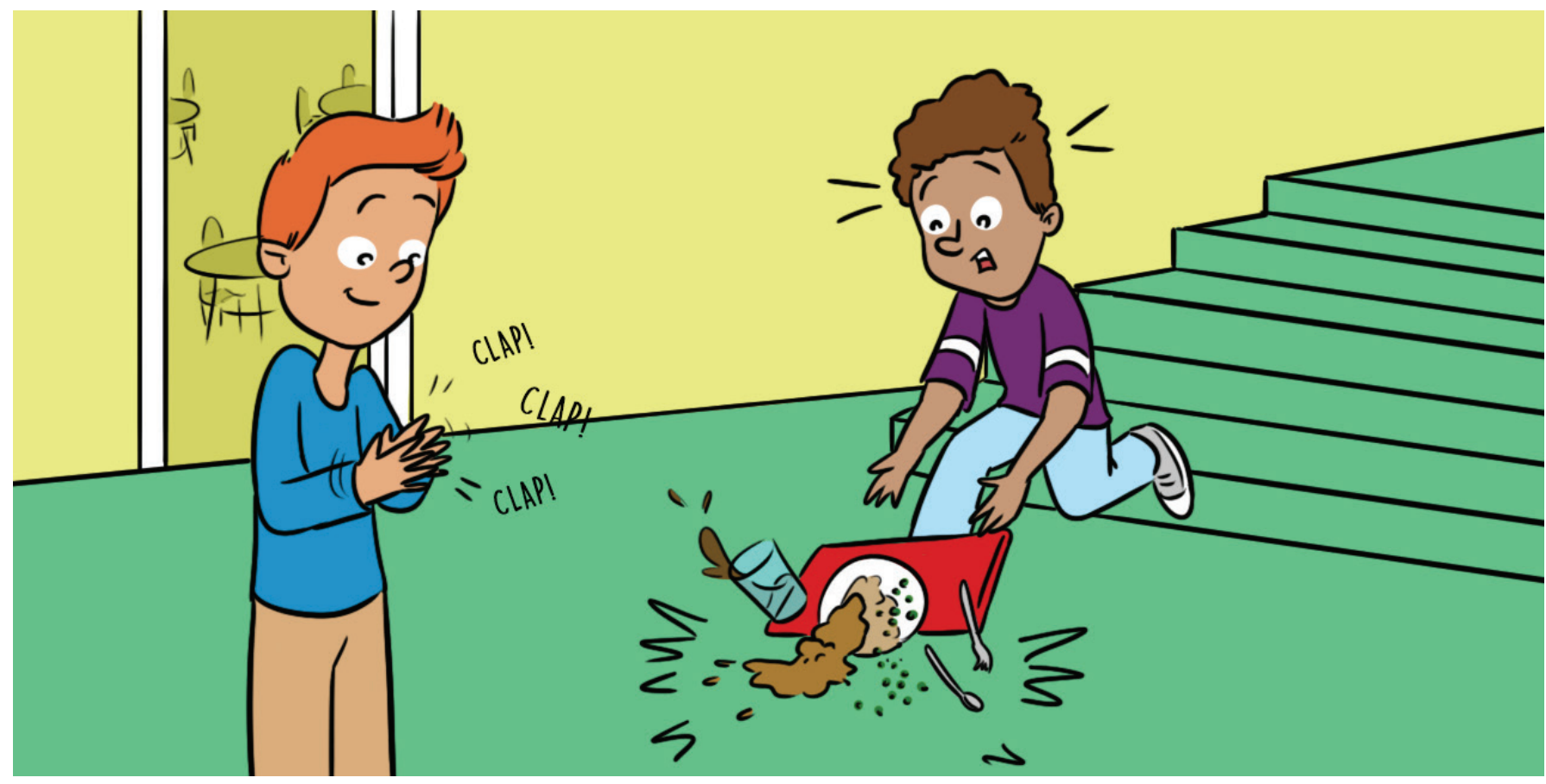

\title{
HOW DO WE UNDERSTAND SARCASM?
}

\section{Penny M. Pexman*}

Department of Psychology, University of Calgary, Calgary, AB, Canada

\section{YOUNG REVIEWERS:}

iexplora!

EXPLORA

SCIENCE

CENTER AND

CHILDREN'S

MUSEUM

AGES: 8-14
Communicating would be a lot easier if everyone just said what he or she meant. But they do not; sometimes people are sarcastic and actually say the opposite of what they mean. Why do people do this? How do we learn to understand sarcasm? What happens in our brains when we are processing sarcasm? These are the questions addressed in scientific research on sarcasm. Here, I explain some of what we have learned from research on these questions. Understanding sarcasm is a challenge for young children, for individuals with autism spectrum disorders, and for some patients with brain damage. Understanding sarcasm depends on advanced language skills and reasoning about other people's minds, and it is supported by a network of brain regions.

\section{INTRODUCTION}

In Harry Potter and the Half Blood Prince, there is a scene where Harry is leaving the Weasley house and Mrs. Weasley says: "Promise me you will look after yourself...stay out of trouble...." Harry responds: "I always do Mrs. Weasley. I like a quiet life, you know me." Anyone familiar with 


\section{SARCASM}

A form of everyday language in which the speaker says the opposite of what he or she means, typically with a distinct tone of voice, to be critical in a funny way.

\section{Box 1}

Did you know? It is possible to be sarcastic without words.
Harry Potter knows that his life is far from quiet, and so he must not really mean what he is saying. In fact, Harry is being sarcastic.

It seems logical to assume that, when we speak, our main goal is for other people to understand exactly what we mean. It is surprising, then, that we sometimes say the opposite of what we mean, speaking sarcastically as Harry does in his response to Mrs. Weasley. Recently, scientific research has given us some good ideas about why we do this, and about how we understand sarcastic speech.

\section{WHY DO WE USE SARCASM?}

Sarcasm has been part of human language for thousands of years. We often use it to be funny, just as Harry does when he speaks sarcastically to Mrs. Weasley. He is trying to be funny and succeeds in making Mrs. Weasley chuckle. We also use sarcasm to talk about things that have not gone the way we expected: we were hoping for good weather for our baseball tournament and it rained, so we say "Great day for a baseball game!" When these things happen, sarcasm can be one way of pointing it out, or criticizing, and at the same time being funny. Some sarcasm is very familiar; sayings like "Yeah, right" or "Thanks a lot" are often used sarcastically. These familiar forms of sarcasm are the type that children usually use first when they begin using sarcasm, around age four or five. Adults and older children often use more complex and creative forms of sarcasm, like Harry does in the example above. More complex forms of sarcasm usually fit a specific situation and can be a way of hiding criticism from a listener. In addition to age differences, there are also personal differences in the way sarcasm is used: some people use sarcasm a lot and others use it very little (Box 1).

We can communicate a sarcastic idea with emoticons or with gesture. For instance, if a friend falls down the stairs and we clap for them, we are using sarcastic gesture. In one study, we looked at communication in 118 family groups. In each family group, one parent and two children worked together to complete a challenging game [1]. We saw examples of both verbal sarcasm (in 32\% of families) and gestural sarcasm (in $80 \%$ of families), used by parents and by children. In an example of gestural sarcasm, one child gave her sibling a slow pat on the back when her sibling failed on his turn in the game. Some families were very sarcastic, using sarcasm many times, and others did not use it at all. Family members seemed to "catch" sarcasm from each other: the use of sarcasm by one member of the family was related to use by others in the family.

Box 1 


\section{WHAT IF WE MISS THE SARCASM?}

Most adults hear sarcastic speech every day and understand it without much difficulty. To understand sarcasm, they rely on the speaker's tone of voice, facial expression, and their knowledge of what the speaker is referring to and how the speaker might feel about it. However, some people struggle to understand sarcasm and tend to think the speaker literally means what he or she has said. The literal meaning is the actual, dictionary meaning of the words used. If you do not understand the sarcasm in what a person says, you miss the joke and may feel left out of the conversation. This can lead to some difficult social situations.

\section{WHEN DO CHILDREN "GET" SARCASM?}

In research in my laboratory, we have found that young children do not usually understand sarcasm until they are 5-6 years old, and they may not find sarcasm funny until they are even older. We study how children understand sarcasm by presenting short puppet shows, in which one puppet says something sarcastic to the other puppet; for instance, "That was a great play" in a puppet show about a soccer game, after one puppet kicks the ball wide of the net and out of bounds. We then ask children a series of simple questions to figure out whether they understood the sarcasm. This work has shown that while 5- to 6-year-old children may understand that the speaker means the opposite of what he or she has said, the children do not understand why the speaker would talk that way; they do not see the humor [2]. Children start to see the humor in sarcasm around 8 or 9 years of age. At younger ages, the jokes that children find funny usually involve unexpected words ("How did the farmer fix his jeans? With a cabbage patch!") or situations (like falling off a trampoline). Around 9 years of age children start to find more humor in teasing other people and also in sarcasm.

In another version of our puppet show task, children tell us what they think the speaker means without having to say much at all. Instead, they show us what the speaker means by choosing one of two objects and putting the object in an answer box (Video available online at: http://fron.tiers.in/go/Gwc32Y). Children are trained in advance that, if they think the puppet has said something nice, they should pick up the "nice duck" and put it in the answer box, and if they think the puppet has said something mean, they should pick up the "mean shark" and put it in the answer box. So, if the children choose the duck, they are showing us that they think the speaker means something nice (the literal meaning). If they choose the shark, they are showing us they think the speaker is being mean (the sarcastic meaning). At the beginning of each experiment, the shark and duck are placed on the table, 


\section{EYE TRACKING}

A research method in which a camera records the position of a person's eyes to measure what they are looking at. Based on eye position, researchers can tell what a person is thinking about while they perform a task. one to the left and one to the right of the child. We put a video camera nearby to continuously record where the children are looking and how long they take when they are making their decisions. Studying where people are looking when they perform a task is called eye tracking. Eye tracking gives researchers clues about what is happening in the brain, because scientists have shown that people tend to look at whatever it is they are thinking about. If children look at the shark while they are making their decision, we assume that they are thinking about the shark (the sarcastic meaning). If children look at the duck while they are making their decision, we assume that they are thinking about the duck (the literal meaning). After selecting the shark or the duck, children are asked two simple questions about what the speaker believed and whether the speaker was trying to be funny. With this experiment, children can demonstrate their understanding of sarcasm without having to explain themselves. This makes this experiment a good choice for use with young children, who are just developing their language skills, and for children with an autism spectrum disorder, who tend to struggle with explaining what people mean.

Using this shark/duck experiment, we were able to test two possible ways to explain how children might process sarcastic language [3]. One theory, called the literal first account, suggests that children must first think about the literal meaning before they can figure out that the literal meaning does not fit, then they move on to the sarcastic meaning. According to this theory, in our experiment, children would look first at the literal response object (the duck) before shifting gaze to the sarcastic response object (the shark). The other theory, called the interactive account, suggests that children do not need to think about the literal meaning first, because they can think about the sarcastic meaning as soon as they hear the puppet's words. According to this theory, in our experiment, children would not necessarily look at the duck first. Our results showed that even 5 -year-old children, who are just beginning to understand sarcasm, tend to look at the shark first (not the duck) when they hear the puppet's sarcastic words. They look at the shark first $85 \%$ of the time. These results are better explained using the interactive account, meaning that the children do not need to think about the literal meaning of the puppet's words before they think about the sarcastic meaning.

We also used this shark/duck procedure in a different study with 31 8-year-old children. First, we measured the children's empathy skills. Empathy is a person's ability to be sensitive to the thoughts and feelings of others. We found that children who had higher scores on the empathy test were more likely to detect sarcasm, and also to look at the shark first in our shark/duck experiment [4]. These findings show that being empathetic and able to understand what is going on in other people's minds may be important for understanding sarcastic speech. 
AUTISM SPECTRUM

\section{DISORDERS}

A group of brain development disorders that affect a person's language abilities and interactions with other people.

MAGNETIC

RESONANCE IMAGING (MRI)

A method used to form pictures of the internal structures of the human body, including the human brain.

\section{DO PEOPLE WITH AN AUTISM SPECTRUM DISORDER UNDERSTAND SARCASM?}

People with an autism spectrum disorder usually have a hard time understanding sarcasm and may take sarcastic speech literally. This makes it hard for them to understand teasing and joking. We used the shark/duck experiment in a study with a group of 19 children with autism spectrum disorders [5]. We compared the results from this group of children with the results from a group of 19 children who did not have autism spectrum disorders. We found that the children with autism spectrum disorders were just as able to detect sarcasm as the children without these disorders. The children with autism spectrum disorders made their choices more quickly: they took on average $3.56 \mathrm{~s}$ to put the shark in the answer box, compared with $4.34 \mathrm{~s}$ for the non-autistic group. Although they were accurate and fast, the children with autism spectrum disorders did not detect that the sarcastic puppet was trying to be funny, while the other children did. This study shows that children who have an autism spectrum disorder can sometimes understand sarcastic speech. This may be because the examples of sarcasm we used in our experiment were quite simple, and the children did not have to explain themselves in words to show their understanding. Instead, they just had to pick the correct object.

\section{WHAT HAPPENS IN THE BRAIN WHEN WE HEAR SARCASM?}

Sarcastic speech can also be challenging for people who have had brain injuries. These people may lose the ability to understand sarcasm after the injury, especially when the injury causes damage to the right half of the brain (also called the right hemisphere). When the damage is to the left hemisphere, difficulties understanding sarcasm tend to be less severe [6]. In a recent study, researchers in Baltimore [7] studied the sarcasm understanding of 24 adults who had suffered a stroke. In a stroke, blood flow to a part of the brain is cut off and the brain cells in that area die. All of the patients had strokes that affected the right hemisphere of the brain. The researchers used an imaging technique called magnetic resonance imaging (MRI) to pinpoint exactly where the stroke had damaged the brain. The researchers also gave the patients a test to see how well they understood sarcasm. The patients were asked to read simple pairs of sentences that had either a sarcastic tone of voice or a non-sarcastic tone of voice, and the patients judged which sentences were sarcastic. For example, "This new show is not bad. It is complete rubbish." They found that the patients who had the most difficulty with the sarcasm test also tended to have more extensive damage in a part of the brain called the right sagittal stratum (Figure 1, pronounced saj-ih-tahl strah-tum). The sagittal stratum is a bundle of 
Figure 1

In this figure, you are viewing the brain as if you were looking at the person's face. The right sagittal stratum is shown in red. This area of the brain is made up of nerve fibers that connect many parts of the right hemisphere. In stroke patients, damage to the area shown here in red is related to problems understanding sarcasm [7].

FUNCTIONAL

MAGNETIC

RESONANCE IMAGING (fMRI)

A form of magnetic resonance imaging that can tell scientists which parts of the brain are active by measuring changes in blood flow to specific brain areas.

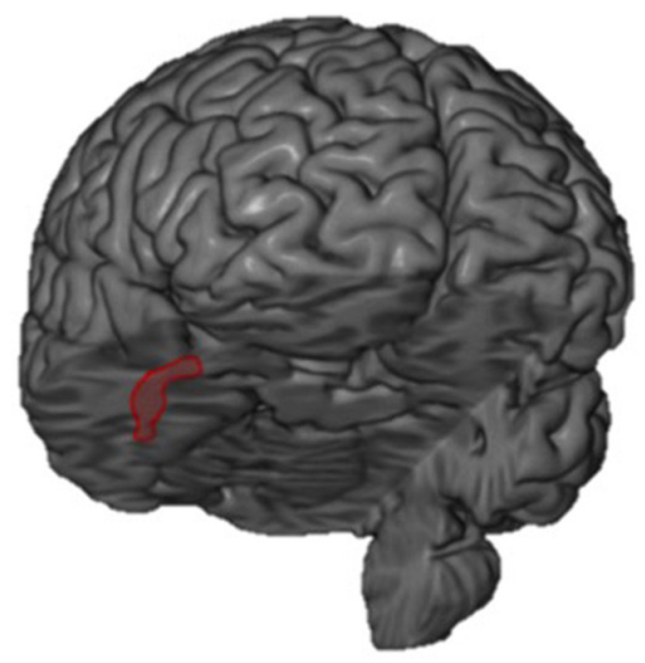

Figure 1

nerve fibers that connects several regions of the brain that help with processing of information that helps people to understand sarcasmboth visual information, like facial expressions, and sounds, like tone of voice. The researchers concluded that the right sagittal stratum must be important for understanding sarcasm.

Other research suggests that many brain areas besides the right sagittal stratum are involved in understanding sarcasm. A group of researchers in France [8] presented 21 healthy adults with sarcastic and literal speech. The adults decided whether each example was sarcastic or literal, while having their brain activity measured with a type of MRI called functional magnetic resonance imaging (fMRI). The researchers found that understanding sarcasm was related to activity in several brain regions, including the left inferior frontal gyrus and the right inferior frontal gyrus (Figure 2). The areas that were found to be involved in understanding sarcasm included areas on the left side of the brain that are involved in understanding language in general, and areas on the right side of the brain that are involved in understanding other people's mental states and recognizing that something is funny.

Both of these imaging studies gave us important information about brain areas linked to understanding sarcasm. We do not yet know how these areas might work together to create a full understanding of sarcasm. In addition, the studies described here used very simple sarcasm tasks, and a goal for future research is to identify the brain networks that support understanding of statements like Harry's, in his conversation with Mrs. Weasley, where context and knowledge of earlier events are important for sarcasm detection. Researchers are working to answer many of these questions using the kinds of tasks and methods I have described in this article. In future research on this topic, it will be 


\section{Figure 2}

In this figure, you are viewing the brain from the left side (left panel) and from the right side (right panel). The left and right inferior frontal gyri are shown in blue. These brain areas were seen to be more active when participants listened to sarcastic statements than when they listened to literal statements [8], although other areas of the brain are also involved.
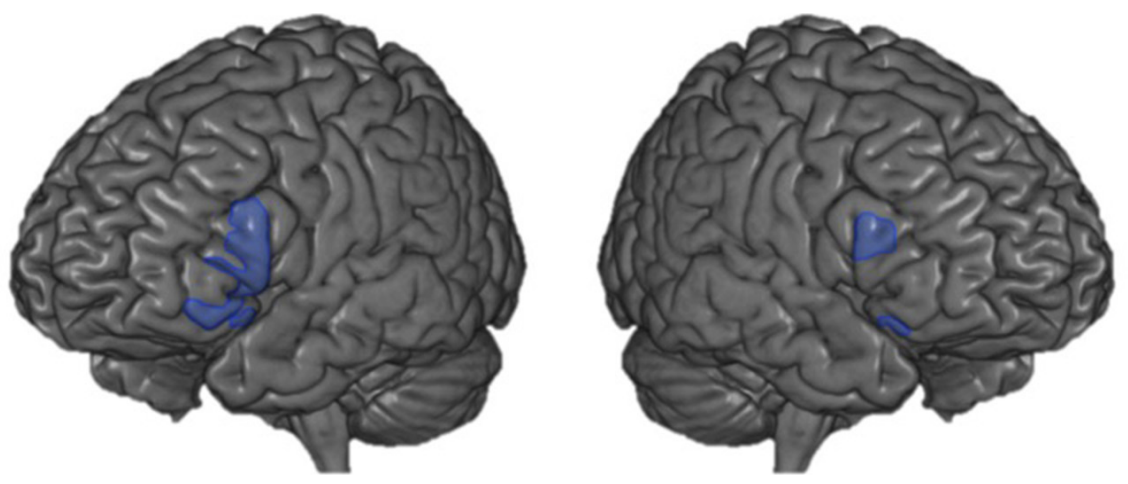

Figure 2

important to figure out whether understanding sarcasm can be taught, and what the best method of training might be, so we can help children and others who struggle to understand sarcasm when it is used in everyday speech.

\section{REFERENCES}

1. Pexman, P. M., Zdrazilova, L., McConnachie, D., Deater-Deckard, K., and Petrill, S. A. 2009. "That was smooth, mom": children's production of verbal and gestural irony. Metaphor Symbol 24:237-48. doi: 10.1080/10926480903310286

2. Harris, M., and Pexman, P. M. 2003. Children's perceptions of the social functions of verbal irony. Discourse Process. 36:147-65. doi: 10.1207/S15326950DP3603_1

3. Climie, E. A., and Pexman, P. M. 2008. Eye gaze provides a window on children's understanding of verbal irony. J. Cogn. Dev. 9:257-85. doi: 10.1080/ 15248370802247939

4. Nicholson, A., Whalen, J. M., and Pexman, P. M. 2013. Children's processing of emotion in ironic language. Front. Dev. Psychol. 4:691. doi: 10.3389/ fpsyg.2013.00691

5. Pexman, P. M., Rostad, K. R., McMorris, C. A., Climie, E. A., Stowkowy, J., and Glenwright, M. R. 2011. Processing of ironic language in children with High Functioning Autism Spectrum Disorder. J. Autism Dev. Disord. 41:1097-112. doi: 10.1007/s10803-010-1131-7

6. Shamay-Tsoory, S. G., Tomer, R., Berger, B. D., Goldsher, D., and Aharon-Peretz, J. 2005. Impaired "affective theory of mind" is associated with right ventromedial prefrontal damage. Cogn. Behav. Neurol. 18:55-67. doi: 10.1097/01.wnn.0000152228.90129.99

7. Davis, C. L., Oishi, K., Faria, A. V., Hsu, J., Gomez, Y., Mori, S., et al. 2016. White matter tracts critical for recognition of sarcasm. Neurocase 22:22-9. doi: 10.1080/13554794.2015.1024137

8. Obert, A., Gierski, F., Calmus, A., Flucher, A., Portefaix, C., Pierot, L., et al. 2016. Neural correlates of contrast and humor: processing common features of verbal irony. PLOS ONE 11: e0166704. doi: 10.1371/journal.pone.0166704 
SUBMITTED: 09 March 2018; ACCEPTED: 26 September 2018;

PUBLISHED ONLINE: 09 November 2018.

EDITED BY: Kathleen Y. Haaland, University of New Mexico, United States

CITATION: Pexman PM (2018) How Do We Understand Sarcasm? Front. Young Minds 6:56. doi:10.3389/frym.2018.00056

CONFLICT OF INTEREST STATEMENT: The author declares that the research was conducted in the absence of any commercial or financial relationships that could be construed as a potential conflict of interest.

COPYRIGHT @ 2018 Pexman. This is an open-access article distributed under the terms of the Creative Commons Attribution License (CC BY). The use, distribution or reproduction in other forums is permitted, provided the original author(s) and the copyright owner(s) are credited and that the original publication in this journal is cited, in accordance with accepted academic practice. No use, distribution or reproduction is permitted which does not comply with these terms.

\section{YOUNG REVIEWERS}

\section{EXPLORA SCIENCE CENTER AND CHILDREN'S MUSEUM, AGES: 8-14}

The Explora Young Minds reviewers are a group of science enthusiasts working with museum educators and mentors from the University of New Mexico. We enjoy learning about the brain through the articles. We also enjoy asking questions and making suggestions to help the scientists make their work more understandable for everyone! We were helped by our Science Mentor Jennifer Walter, who just received her PhD in pediatric neuropsychology. she enjoys working with kids, playing with her dog, and tries to cook new recipes.

\section{AUTHOR}

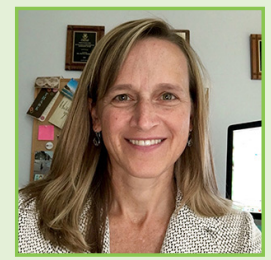

\section{PENNY M. PEXMAN}

I am a Professor of Psychology at the University of Calgary, Canada. I am a cognitive scientist and I study how we understand language. I use lots of different methods in my research, including eye tracking and brain imaging. When studying what children understand about language I use fun tasks and puppet shows so my lab is home to a large collection of puppets and props. I first started studying the psychology of sarcasm when I was an undergraduate student, over 25 years ago, and it has been an important part of my research program ever since. *pexman@ucalgary.ca 